\title{
On certain approaches to the control methods development for the precipitation formation processes in convective clouds
}

\author{
Vitaly A. Shapovalov ${ }^{1}$, Ludmila M. Fedchenko ${ }^{1}$, Boris P. Koloskov ${ }^{2}$, \\ Aleksey A. Bychkov ${ }^{2 *}$, Boris A. Ashabokov ${ }^{1}$, Alexander V. Shapovalov ${ }^{1}$ \\ ${ }^{1}$ Federal State Budgetary Institution "High Mountain Geophysical Institute" (FSBI "HMGI") \\ Federal Service for Hydrometeorology and Environmental Monitoring), 2 Lenin av., Nalchik, 360030, Russia \\ ${ }^{2}$ Autonomous Nonprofit Organization "Agency of Atmospheric Technologies", office 204, \\ 15 Druzhinnikovskaya street, Moscow, 123242, Russia \\ *e-mail: a.bychkov81@mail.ru
}

Received: 18 August 2018 / Accepted: 12 August 2019

\begin{abstract}
The article aims at searching for the optimal way of emission of ice nucleating agent in convective cloud in order to prevent formation of harmful hail by analyzing simulations of this process within a numerical model of cloud!. The state of the physics of clouds and active influences on them is discussed. It is noted that at the present time studies of the regularities of the formation and development of clouds as a whole begin taking into account their systemic properties. The main directions of research at the next stage of its development are discussed. The features of the existing methods of active action on convective clouds are noted, the main tasks encountered in the development of methods for controlling sedimentation in convective clouds by introducing reagents are formulated. It is noted that research on the development of methods for active influence on clouds should be conducted on the basis of new and more effective approaches, which should be based on the extensive use of mathematical modeling. Some approaches to solving this problem are discussed. According to the authors, the most promising of them are approaches based on the theory of optimal control and bifurcation theory. Some results of numerical modeling of the active effect on convective clouds are given.
\end{abstract}

Keywords: physics of clouds, convective clouds, hail formation, multidimensional models of clouds, artificial modification of convective clouds, optimal control, bifurcation theory.

\section{Introduction}

The North Caucasus and southern Russia are the most prone to natural disasters due to geographical features. More than fifty years ago, a number of paramilitary antihail services were created in the USSR, including three in the North Caucasus - the Krasnodar, North Caucasus and later Stavropol services - which protect an area of 2.65 million hectares in the North Caucasus and Southern Federal counties.
The status of existing technology of active impact on the precipitation formation processes in clouds is complex and ambiguous. It is still based on the concepts suggested in 1950s-60s, rather than on methods developed as a result of precise studies of cloud behavior under active impacts. An important note is that despite the state of this technology, there is an extensive work on the active impact on clouds (Stephens et al., 2019; UCP, 2019).

It is important to mention that cloud physics proceeds to the next development stage in a natural way, as beside 
individual basic processes, clouds have numerous factors that impact cloud formation and development. They include factors whose impact on the formation and development of clouds have been studied insufficiently, as well as factors whose role in the cloud and precipitation formation is not completely clear.

\section{Study area}

An analysis of the current state of the physics of clouds and active impacts on them in (Ashabokov et al., 2014; 2018) showed that this scientific line has slowed down its development in recent decades. The publications' authors noted that it is due to the current gradual shift to the study of regularities in cloud formation and development as a whole with account of their system properties.

Publications (Bychkov \& Shapovalov, 2017; Ashabokov et al., 2018) approached convective clouds as sophisticated physical systems and identified factors that form the structure of clouds. These are process interaction in clouds (the emergent system properties) and "cloud-atmosphere" interaction (the hierarchy property).

The study result aggregation at the last stage of the cloud physics development has achieved noticeable success: multidimensional models of clouds have been developed, as well as models with detailed processes, which can be used for this purpose (Ashabokov et al., 2014; 2018; Ashabokov \& Shapovalov, 2008; Veremei et al., 2016; Vladimirov \& Pastushkov, 2016; Dovgaluk et al., 2016; Drofa, 2010; Clark, 1979; Cotton et al., 1982; Farley, 1987; Orville \& Kopp, 1977). Publications (Ashabokov et al., 2018; Dovgaluk et al., 2016; Kogan et al., 1984; Shmeter, 1987; Straka, 2009) offer an inclusive review of publications on the numerical cloud simulation. A number of numerical studies have been conducted also on the effects of seeding (Chae et al., 2018; Curic et al., 2019). We will limit ourselves to only noting that achievements in this domain make it possible to obtain significant fundamental and applied results.

Obtaining a new knowledge on the regularities of formation and development of clouds and developing effective and scientifically justified methods for active impact on clouds have become very relevant problems of the physics of clouds and impacts on them (Vladimirov \& Pastushkov, 2016; Drofa, 2010; Koloskov, Korneev \& Shchukin, 2012; Pastushkov, 2016; Cotton et al., 1982; Farley, 1987). In this connection, the subsequent stages of its development will be focused on the study of regularities of the macro and micro structural characteristics of clouds and development of new approaches to modeling active impacts on them.

Some publications studying the role of the above factors in the cloud and precipitation formation processes have al- ready been released. For example, publications (Ashabokov et al., 2014; Bychkov \& Shapovalov, 2017; Shapovalov et al., 2018) provide the simulation results based on a 3D model of convective clouds that comprehensively accounts for the wind field structure impact processes on the cloud and precipitation formation processes in the atmosphere. As for the role of the interaction of cloud formation and development processes, special models need to be developed for its study. Publication (Ashabokov et al., 1994) deals with the problem and studies the impact of the thermodynamic parameter fields' deformation in clouds (as a result of process interaction in clouds) on the formation of their microstructural characteristics.

Our publication considers the main problems of the development of methods for the precipitation formation process control as regards to convective clouds and presents several solutions of this problem. It is important to note that the development of methodology and methods for the solution of the problem of active impact on convective clouds, which is connected with the study of the behavior of a very sophisticated dynamic system (convective cloud) for various options of reactive chemical application, has not yet been paid due attention.

\section{Materials and methods}

\subsection{Approaches to the development of methods for active impact on convective clouds}

Considering the existing approaches to the development of methods for active impact on convective clouds, the most popular method is the simulation of various options of application of the reactive chemical particles in a cloud and selecting the most effective one in terms of achieving the impact goal.

The reactive chemical input options can vary by the parameters of the added reactive chemical particles' source. Depending on the method for the reactive chemical application in a cloud, the source can be of a point or linear nature or be more complex. Note that very diverse models of clouds are used in the study based on this approach (Veremei et al., 2016; Vladimirov \& Pastushkov, 2016; Dovgaluk et al., 2016; Drofa, 2010; Kogan et al., 1984; Clark, 1979; Cotton et al., 1982; Farley, 1987; Orville \& Kopp, 1977).

\subsection{The goals to achieve}

- find a local region in the cloud, which has the most favorable conditions for active impact to achieve the goal;

- identify the reactive particle concentration that should be achieved in such a region at each time 
point, i.e. identify the reactive chemical application dynamics.

Obviously, solving these problems based on certain assumptions and admissions regarding the process of precipitation formation in clouds, as is done in the existing concepts, is deemed impossible. Any approaches to their solution should be based on using numerical cloud models. However, it is also important to keep in mind that the simulation of various options of reactive chemical application in clouds provides an approximate solution only. Especially as regards to the second problem.

\section{Results and Discussion}

\subsection{Results of simulating an active impact on a hail cloud based on a $2 \mathrm{D}$ model}

Let us consider in more details some of the results of the simulation of the impact of various options of artificial crystal application in a cloud on the risk of hail formation. The said results show that the effect of the impact on the cloud is sophisticated and depends on many factors. These options differed by the area of application and the concentration of artificial crystals in this area. To estimate the risk of hail formation in the cloud, we used the number of large-size hail stones emerging in the cloud during its evolution. The calculation used a 2D model of hail clouds (Ashabokov et al., 1994, 2018).

Table 1 provides the calculation results for the case of distribution of the same number of artificial crystals $\left(3 \times 10^{13}\right)$ in each element volume $(500 \mathrm{~m} \times 250 \mathrm{~m})$ of the application area during the impact period. The shaded element volume in the options under consideration is located on the isotherm $-10^{\circ} \mathrm{C}$ in the area of moderate updraft.

It is obvious from Table 1 that an increase in the application area (options 2-6) changes the seeding effect insignificantly compared to option 1 . This proves that the emergence of large-size hail stones can be prevented by only distributing crystals in the part of the cloud where the conditions are favorable for this purpose. It follows that the development of methods for the control of precipitation formation in convective clouds requires solving the first task (finding the local region in the cloud where the conditions are favorable for active impact to achieve the goal).

The hail formation risk in Table 1 in the clouds is determined by the max size and intensity of hail, areas of hailstorms, degree of damage and losses from hail. Seeding effect shows how many percent the number of hail particles falling out of the cloud has decreased.

The calculation results also reveal the importance of determining the reactive particle concentration that needs to be reached in the region of application to prevent the formation of hazardous hail stones in the cloud.
Table 1. Seeding effect depending on volume and geometry of crystallizing reagent application area

\begin{tabular}{|c|c|c|c|c|}
\hline № & Source form & $\begin{array}{c}\text { Source } \\
\text { intensity } \\
\left(\mathrm{m}^{-3} \mathrm{~s}^{-1}\right)\end{array}$ & $\begin{array}{c}\text { Risk } \\
\text { of hail } \\
\text { formation } \\
(\%)\end{array}$ & $\begin{array}{c}\text { Seeding } \\
\text { ef } \\
\text { fect } \\
(\%)\end{array}$ \\
\hline 1 & . & $10^{6}$ & 14.8 & 85.2 \\
\hline 2 & $\square$ & $10^{6}$ & 8.2 & 91.8 \\
\hline 3 & \begin{tabular}{|l|l|l|l|} 
& & - & \\
\end{tabular} & $10^{6}$ & 4.5 & 95.5 \\
\hline 4 & 正 & $10^{6}$ & 8.3 & 91.7 \\
\hline 5 & \begin{tabular}{l|l} 
\\
\end{tabular} & $10^{6}$ & 8.1 & 91.9 \\
\hline 6 & $\square$ & $10^{6}$ & 10.1 & 89.9 \\
\hline
\end{tabular}

Figure 1 shows the results of simulating an active impact on a hail cloud by applying artificial crystals in it. We considered the cases when the crystal source was located at various points within the cloud. The calculation was based on a 2D model of microphysical processes in hail clouds with given thermodynamic properties.

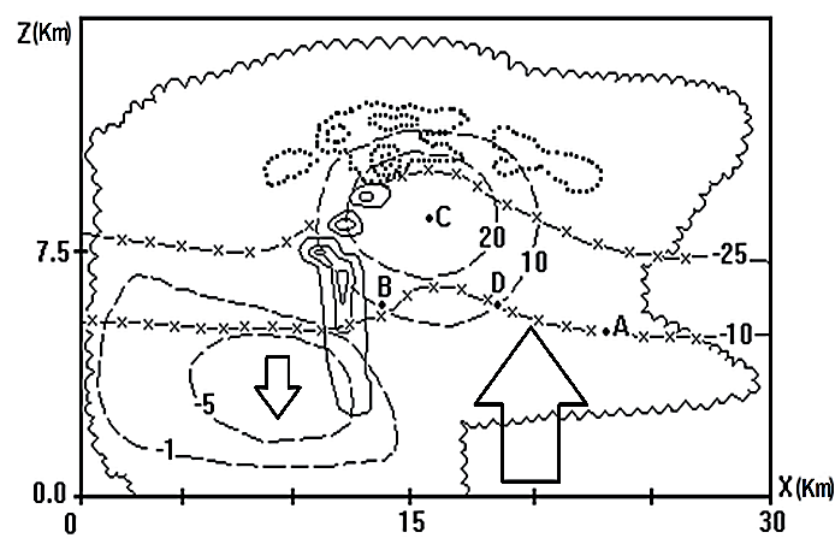

Figure 1. Cloud structure for time $\mathrm{t}=18 \mathrm{~min}$ :

isolines of concentration of large hailstones $[\mathrm{r} \geq 1 \mathrm{~cm}]$; ......... isolines the concentrations of crystals with $\mathrm{d}=2-5 \mathrm{~mm}$; $-\mathrm{x}-\mathrm{x}-\mathrm{x}-$ temperature isolines corresponding to $-10^{\circ} \mathrm{C}$ and $-25^{\circ} \mathrm{C}$; ----- isolines of vertical flow velocity

The results showed that an impact with a spot source in the region with moderate upward streams (point A in Fig.1) leads to a decrease in the number of large-size hail stones forming in the cloud during its evolution (approximately by $30 \%$ compared to their number in case of no impact). In case 
the source of artificial crystals was in the region marked by point $\mathrm{B}$, we observed a significant decrease in the total number of large hail stones, approximately by $60 \%$.

The most effective option in terms of the cloud's hail formation risk mitigation was the one with the source located in point $\mathrm{D}$, which is at isotherm $-10^{\circ} \mathrm{C}$ in the region of moderate updraft. Moving the source of artificial crystals to this point resulted in the $90 \%$ decrease of the hail formation risk in the cloud.

In case the source was placed at point $C$, we observed an increase in the number of formed large hail stones compared to no impact, i.e. the impact results in a negative effect. The physics of the effect of artificial ice nuclei on the cloud microstructure in point $\mathrm{D}$ is that the effect in the central part of the cloud, near the ascending flow, by small artificial crystals at a concentration of $10^{6} \mathrm{~m}^{-3}$ results in the partial or complete elimination of the liquid droplet there, resulting in a number of large hail decreases.

\subsection{Results of simulating an active impact on the hail cloud based on a 3D model}

Consider some of the results of simulating an active impact on the hail cloud, based on a 3D model with detailed reproduction of thermohydrodynamic and microphysical processes

Figure 2 shows vertical sections of the cloud at the same time point (40 min), which correspond to its development in natural conditions and under an active impact. Figure 2a shows a cloud developing in natural conditions, while Figure $2 \mathrm{~b}$ shows a cloud with applied crystallizing reactive chemical at the $30^{\text {th }}$ minute of development. It is obvious that the active impact significantly changed the microstructural characteristics and radar reflectivity factor of the cloud in the area of artificial crystal application. Fig- ure 2 shows that the area of large-size ice particles almost perished as a result of application.

This approach to the development of methods for precipitation formation control in clouds, based on the selection of various options of reactive chemical distribution, is not perfect. One of its drawbacks is the need to use smallsize spatial grids in calculation, as in case of its large size, the cloud region where the reactive particles should be distributed, can remain unnoticed. Another issue is related to the changing intensity of the reactive chemical distribution in the cloud over time. This difficulty will be especially sensible in case of a small size of the cloud region to distribute reactive chemicals for the precipitation formation control purposes (clouds in arid and semiarid areas).

\subsection{Approach to simulation of an active impact on convective clouds in terms of the optimal control theory}

A more promising method for cloud precipitation formation control is to consider the problem in terms of the optimal control theory (Pontryagin et al., 1976). This approach is implemented in the case of hail clouds (the problem of preventing the emergence of hazardous hail stones in clouds) (Ashabokov et al., 2014; Ashabokov \& Shapovalov, 1996).

As is known, the solution of optimal control problems is always hindered by serious difficulties. These difficulties do not allow to study the possibility to control the cloud microstructure formation using certain control models, which have been recorded based on a sufficiently complete cloud models. Therefore, the problem of optimal control of hail cloud evolution in these publications was considered for a system of equations describing microphysical processes in the clouds against the background of the given thermohydrodynamics (Ashabokov et al., 2013):

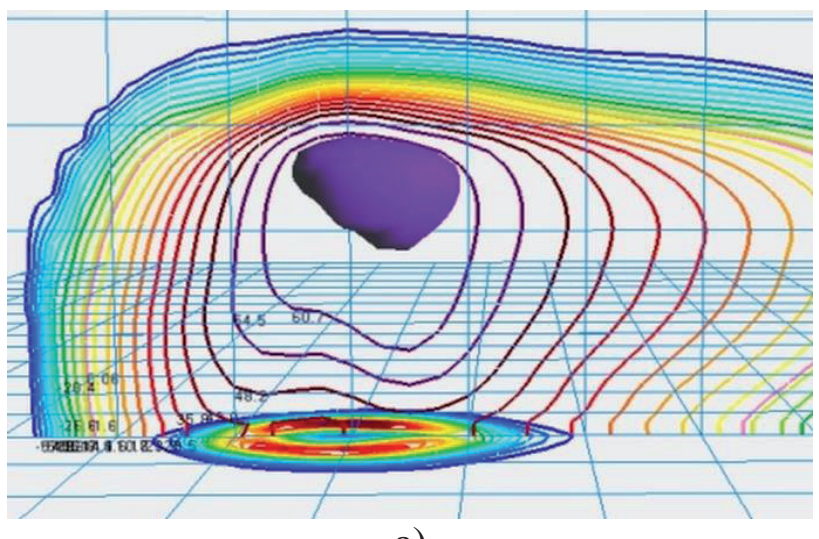

a)

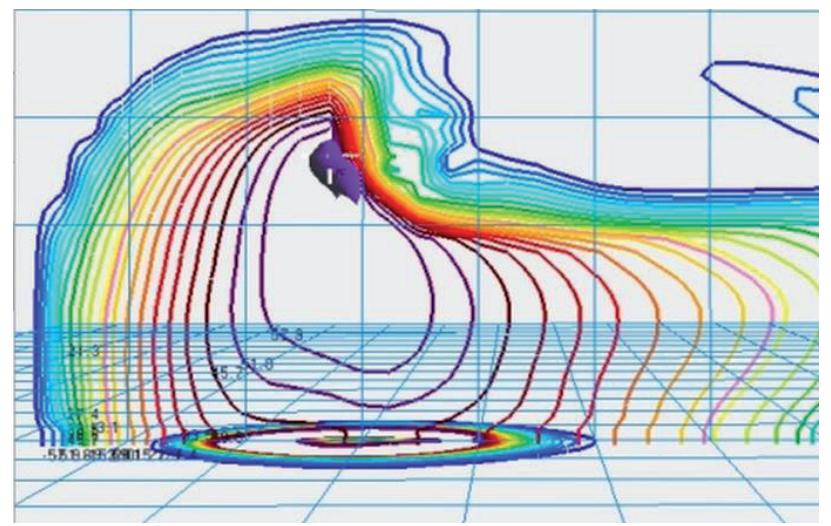

b)

Figure 2. Isolines of radar reflectivity (dBZ) during natural cloud evolution (a) and when seeding with crystallizing reagent (b). Inside the cloud is a zone of localization large ice particles. Figures a, b show additional horizontal and vertical auxiliary grids with $2 \times 2 \mathrm{~km}$ cells. 


$$
\begin{aligned}
& \frac{\partial f_{1}}{\partial t}+V_{x} \frac{\partial f_{1}}{\partial x}+\left(V_{z}-V_{1}\right) \frac{\partial f_{1}}{\partial z}=\left[\frac{\partial f_{1}}{\partial t}\right]_{K}+\left[\frac{\partial f_{1}}{\partial t}\right]_{a}+\left[\frac{\partial f_{1}}{\partial t}\right]_{\partial}+\left[\frac{\partial f_{1}}{\partial t}\right]_{3}+\frac{\partial}{\partial x} K \frac{\partial f_{1}}{\partial x}+\frac{\partial}{\partial z} K \frac{\partial f_{1}}{\partial z}+I_{1} \\
& \frac{\partial f_{2}}{\partial t}+V_{x} \frac{\partial f_{2}}{\partial x}+\left(V_{z}-V_{2}\right) \frac{\partial f_{2}}{\partial z}=\left[\frac{\partial f_{2}}{\partial t}\right]_{a}+\left[\frac{\partial f_{2}}{\partial t}\right]_{3}+\frac{\partial}{\partial x} K \frac{\partial f_{2}}{\partial x}+\frac{\partial}{\partial z} K \frac{\partial f_{2}}{\partial z}+I_{2}+u
\end{aligned}
$$

$0 \leq x \leq L_{x}, 0 \leq z \leq L_{z}, 0 \leq m<\infty, t>0$,

where $f_{1}(x, z, m, t)$ and $f_{2}(x, z, m, t)$ are drop and ice particle mass spectrum in the cloud at time point $t$ at point $(x, z)$. Summands $I_{1}(x, z, m, t)$ and $I_{2}(x, z, m, t)$ in the right parts of the equations describe the drop and crystal sources in natural conditions, $u(x, z, m, t)$ is the source of artificial crystals. The cloud's microstructure evolution is controlled by including the source of artificial crystals. The rest summands in the right parts of the equations describe the change in function $f_{1}(x, z, m, t)$ by the processes of drop coagulation, accretion, fractioning, and freezing, function $f_{2}(x, z, m, t)$ by accretion and freezing of overcooled drops, as well as the change in these functions due to the turbulent transport. Turbulent intensity increases with height in deep clouds while remaining approximately constant in shower clouds (Feist et al., 2019).

The following conditions are met at the boundary of the space region:

$$
\begin{aligned}
& f_{1}(x, z, m, t)=f_{2}(x, z, m, t)=0 \\
& \quad \text { or } x=0, x=L_{x}, z=L_{z}, \\
& \partial f_{1}(x, z, m, t) / \partial z=\partial f_{2}(x, z, m) / \partial z=0 \text { for } z=0 .
\end{aligned}
$$

The the drop and ice particle mass spectrum at the initial time point is known

$$
\begin{aligned}
& f_{1}(x, z, m, 0)=f_{1}^{0}(x, z, m) \\
& f_{2}(x, z, m, 0)=f_{2}^{0}(x, z, m)
\end{aligned}
$$

Thus, function $u(x, z, m, t)$ is the control in the problem, describing the source of artificial crystals. Proceeding from the fact that in real situations only a certain part of the cloud is affected by the impact at each time point, we write down $u(x, z, m, t)$ as a point source:

$u(x, z, m, t)=u_{0}(m, t) \cdot \delta\left(x-x_{0}(t)\right) \cdot \delta\left(z-z_{0}(t)\right)$

where $x_{0}(t)$ and $z_{0}(t)$ are the coordinates of the spectrum by axes $O X$ and $O Z, u_{0}(m, t)$ is the intensity of the artificial crystal source, the distribution of which is given, $\delta$ is the delta function. Limitations are obvious for

$$
\begin{aligned}
& u_{0}(m, t), x_{0}(t) \text { and } z_{0}(t): \\
& u_{0}(m, t) \geq 0, \\
& x_{0}(t) \in\left[0, L_{x}\right], \\
& z_{0}(t) \in\left[h_{1}, h_{2}\right],
\end{aligned}
$$

where $h_{1}$ и $h_{2}$ are the upper and lower boundaries of the region, in which the ice-forming activity of the reactive chemical takes place (when $z<h_{l}$, crystals do not emerge due to the high temperature, while at $z>h_{2}$, there are many natural crystals).

Task (1) - (3) with the given control function (4) reproduces in its solution the evolution of the cloud particle system for a specific control type. The process proceeds naturally at $u(x, z, m, t)=0$. The cloud development path depends on control (4), while its quality is determined by the extent of the impact goal achievement. The number of large-size particles emerging in the cloud for the time of evolution $[0, \mathrm{~T}]$ was used as the composite function used for estimating the impact goal achievement:

$$
F[\mathrm{u}(\mathrm{x}, \mathrm{z}, \mathrm{m}, \mathrm{t})]=\int_{0}^{L_{L}} \int_{0}^{L_{L}} \int_{m_{k}}^{\infty} \int_{0}^{T} f_{2}(x, z, m, t) d x d z d m d t
$$

where $m_{k}$ is the mass of a hail stone that reaches the ground and able to cause any damage.

In this case, the control function $u(x, z, m, t)$ is included in the right part of the expression for the distribution function of ice particles by masses $f_{2}(x, z, m, t)$, and affects the change of this parameter with time. Changing the function $u(x, z, m, t)$ we obtain the change $f_{2}(x, z, m, t)$ on the phase trajectory of the cloud particle system, and as a result the integral index $\mathrm{F}[\mathrm{u}]$ changes.

In this case, the problem of optimal control of the cloud microstructure is formulated as follows: find the admissible form of control function (4), which brings a minimum to composite function (5), for controlled system (1)-(3):

$$
F[u] \rightarrow \min .
$$

This approach allows studying the behavior of function $\mathrm{u}(\mathrm{x}, \mathrm{z}, \mathrm{m}, \mathrm{t})$ over time. As cloud particle distribution functions $f_{1}(x, z, m, t)$ and $f_{2}(x, z, m, t)$ describing the state of the distribution object (the cloud microstructure), and control function $u(x, z, m, t)$ are of distributed nature, we have a problem of optimal control with distributed parameters (Pontryagin et al., 1976). 
Figure 3 shows the view of optimal control function obtained for a specific hail cloud.
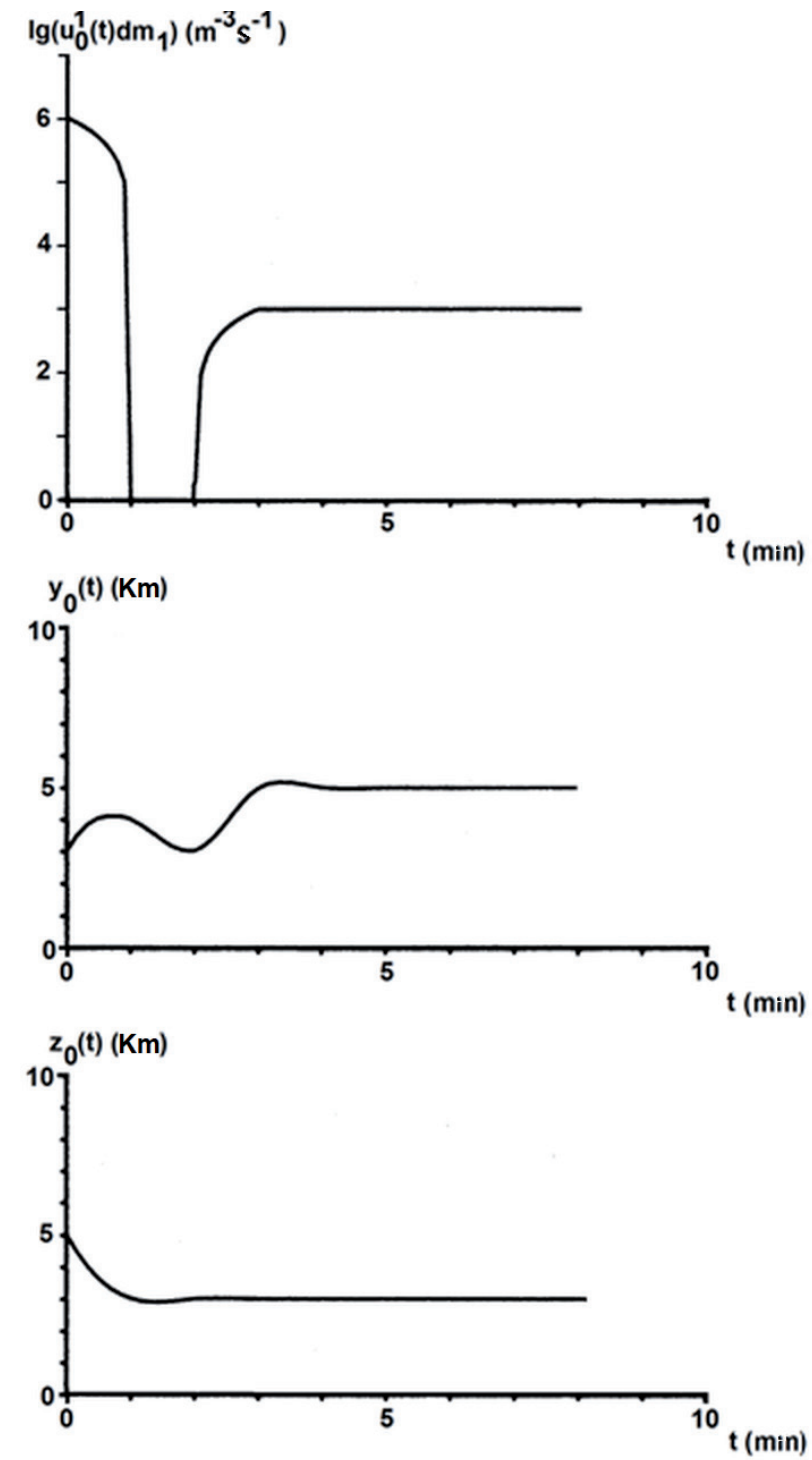

Figure 3. View of optimal control function $u_{0}^{1}(t), y_{0}(t)$, and $z_{0}(t)$

It is obvious that function $u(x, z, m, t)$ changes over time according to a sophisticated pattern. Function $u_{0}(m$, t) (the intensity of the artificial artificial ice nuclei source), for example, decreases quickly from its value at the initial time point and shortly after the impact start almost reaches zero, i.e. the application of artificial crystals is ceased.

Then, after a while, the source intensity starts increasing and after achieving a certain value remains constant. As is seen in the figure, the point coordinates after the application of artificial crystals also change according to a complex pattern after the beginning of active impact. However, they stabilize after a while and remain unchanged over time. Note that the transition of the source of arti- ficial crystals (function $u(x, z, m, t)$ ) into a fixed state after a certain period from the beginning of active impact is due to the peculiar features of the numerical method of the optimal control problem solution (the method of spatial approximations in the control space) (Ashabokov \& Shapovalov, 1996).

For relatively late time points, the view of the control functions remains equal to their initial approximations given in the model. This is an evidence of the fact that the cloud eventually better reacts to an earlier impact, rather than to a later one. In fact, the provided option should have the reactive chemical applied just one minute before the beginning of its impact.

\subsection{Simulation of an active impact on convective clouds based on the bifurcation theory}

The third approach to the development of methods for cloud precipitation formation control, which is being developed by the High Mountain Geophysical Institute currently, is the approach based on the bifurcation theory. In our opinion, this approach can essentially facilitate the solution of this problem. Bifurcation theory tells us that the existence of bifurcation points (he parameter values at which the system's dynamics suddenly changes) is preserved under small deformations of the model equations, but the numerical values may change (Bathiany et al., 2016; Dortmans et al., 2019). Qualitatively there is good reason to believe that the existence of the bifurcation points in the model will be preserved in similar more refined models and in the real world

The problem of development of a method for hail cloud impact can have as a parameter of bifurcation, for example, the source parameters describing its position in the cloud and the intensity of artificial crystal distribution, as well as the parameters of the mass spectrum based on the artificial crystals' size. The problem is solved by finding the values of these parameters (bifurcation parameters), at which the cloud converts from the hail state to non-hail state.

Research aiming to find such bifurcation points is focused on two trends:

(1) these points are found by using the distributed parameter model, i.e. the specified above model of microphysical processes in hail clouds with given thermohydrodynamics.

(2) using the model with concentrated parameters, which is obtained as a result of conversion by the method of Galerkin, used in the first trend of the model of microphysical processes in hail clouds. This approach is better optimized than the former currently. 


\section{Conclusions}

The study allowed us to conclude that the described approaches to the development of control methods for the precipitation formation in clouds are universal, i.e. they can be used for the development of both hail damage prevention and precipitation control methods.

It should be noted that the solution of the optimal control problem (1)-(5) faces more difficulties than a simulation of the cloud formation and development, based on model (1)-(3). However, despite the difficulty in solving problem (1)-(5), optimal control approach is very productive and ensures more promising results. In particular, it allows studying the change in the artificial crystal source intensity over time. The approach based on the bifurcation theory using the model with concentrated parameters is better optimized than the one with finding bifurcation points using the distributed parameter model.

As a result of a comparative analysis of these approaches, we will determine the more effective one and use it for further research and development of methods for the control of precipitation formation in convective clouds. This will be accompanied by a transition to more sophisticated models of clouds, e.g. a 3D model with a comprehensive consideration of processes.

\section{References}

Ashabokov B.A., Fedchenko L.M., Shapovalov A.V. \& Shoranov R.A., 1994, Numerical studies of the formation and growth of hail with the natural development of the cloud and the active influence. Meteorology and Hydrology 1: 41-48.

Ashabokov B.A., Shapovalov A.V., Kuliev D.D., Prodan K.A. \& Shapovalov V.A. 2014, Numerical Simulation of Thermodynamic, Microstructural, and Electric Characteristics of Convective Clouds at the Growth and Mature Stages. Radiophysics and Quantum Electronics 56(11): 811-817. (http://doi.org/10.1007/S11141014-9483-Z).

Ashabokov B.A. \& Shapovalov A.V., 1996, Numerical model of formation of microstructure control hail clouds, Proceedings of the Academy of Sciences. Physics of the Atmosphere and the Ocean 32(3): 364-369.

Ashabokov B.A. \& Shapovalov A.V., 2008, Convective clouds: numerical models and simulation results in natural and active environments. Publishing house KBSC RAS, Nal'chik.

Ashabokov B.A., Fedchenko L.M., Tapashenkhanov V.O., Shapovalov A.V., Shapovalov V.A., Makuashev M.K., Kagermazov A.Kh., Sozaeva L.T., Tashilova A.A. \& Kesheva L.A., 2013, The physics of hail clouds and ac- tive influences on them: state and development trends. Pechatnyj dvor, Nal'chik.

Ashabokov B.A., Fedchenko L.M., Shapovalov A.V., Kalov Kh.M., Kalov R.Kh, Tashilova A.A. \& Shapovalov V.A., 2018, Mathematical Modeling of the Influence of the Wind Field Structure in the Atmosphere on the Cloud Formation Processes. Atmospheric and Climate Sciences 8(1): 84-96. (doi: 10.4236/acs.2018.81006).

Bathiany S., Dijkstra H., Crucifix M., Dakos V., Brovkin V., Williamson M.S., Lenton T. \& Scheffer M., 2016, Beyond bifurcation: using complex models to understand and predict abrupt climate change. Dynamics and Statistics of the Climate System 1(1): dzw004. (https:// doi.org/10.1093/climsys/dzw004).

Bychkov A.A. \& Shapovalov V.A., 2017, Formation of Bulk Electric Charges and Fields during Development of Thunderstorm Clouds, Research India Publications. International Journal of Applied Engineering Research, ISSN 0973-4562 12(23): 13150-13157.

Chae S., Chang K.-H., Seo S., Jeong J.-Y., Kim, B.-J. Kim Ch.K., Yum S.S. \& Kim J., 2018, Numerical Simulations of Airborne Glaciogenic Cloud Seeding Using the WRF Model with the Modified Morrison Scheme over the Pyeongchang Region in the Winter of 2016. Advances in Meteorology, ID 8453460. (https://doi. org/10.1155/2018/8453460).

Clark T., 1979, Numerical simulation with a tree-dimension cloud model: lateral boundary condition experiments and Multiceller severe storm simulations. J. Atm. Sci. 36(11): 2191-2215.

Cotton W.R., Stephens M.A., Nehrkorn T. \& Tripoli G.J, 1982, The Colorado State University three-dimensional cloud/mesoscale Model. Part II: An Ice Phase Parameterization. J. Rech. Atmos. 16: 295-320.

Curic M., Lompar M., Romanic Dj., Zou L. \& Liang H., 2019, Three-Dimensional Modelling of Precipitation Enhancement by Cloud Seeding in Three Different Climate Zones. Atmosphere 10: 294. (doi:10.3390/atmos10060294).

Dortmans B., Langford W.F. \& Willms A.R., 2019, An energy balance model for paleoclimate transitions. Climate of the Past 15: 493-520. (https://doi.org/10.5194/ cp-15-493-2019).

Dovgaluk Yu.A., Veremei N.E., Vladimirov S.A., Drofa A.S., Zatevakhin M.A., Ignatiev A.A., Morozov V.N., Pastushkov R.S., Sinkevich A.A. \& Shapovalov A.V., 2016, The concept of development of numerical nonstationary three-dimensional model of precipitation forming convective cloud in natural conditions and during active modifications. Proceedings of A.I. Voeikov Main Geophysical Observatory 582: 7-44.

Drofa A.S., 2010, Research on effects of hygroscopic particles to warm convective clouds on the numerical mod- 
eling results. Bulletin of Russian Academy of Sciences. FAO 46(3): 357-367.

Farley R.B., 1987, Numerical modeling of hailstone growth. Part III: Simulation of an Alberta hailstorm natural seeded cases. J. Claim, Appl. Met. 26(7): 789812.

Feist M.M., Westbrook C.D., Clark P.A., Stein T.H.M., Lean H.W. \& Stirling, A.J., 2019, Statistics of convective cloud turbulence from a comprehensive turbulence retrieval method for radar observations. Quarterly Journal of the Royal Meteorological Society 145(719): 727744. (doi: https://doi.org/10.1002/qj.3462).

Kogan E.L., Mazin I.P., Sergeev B.N. \& Khvorostyanov V.I., 1984, Numerical modeling of clouds. Gidrometeoizdat, Moscow.

Koloskov B.P., Korneev V.P. \& Shchukin G.G., 2012, Methods and means of modification of clouds, precipitation and fog. RSHU Publishers, St. Petersburg.

Orville R.D. \& Kopp F.J., 1977, Numerical simulation of the life history of a hailstorm. Journal of the Atmospheric Sciences 34(10): 1596-1618. (https://doi. org/10.1175/1520-0469(1977)034<1596:NSOTLH $>2$. $0 . \mathrm{CO} ; 2)$.

Pastushkov R.S., 2016, The model of convective cloud modification with ice-forming aerosols. Present-day status and perspective. Proceedings of A.I. Voeikov Main Geophysical Observatory 582: 128-158.

Pontryagin L.S., Boltyansky V.G., Gamkrelidze R.V. \& Mishchenko E.F., 1976, Mathematical theory of optimal processes. Nauka, Moscow.
Shapovalov V.A., Shapovalov A.V., Koloskov B.P., Kalov R.Kh., Stasenko V.N., 2018, Numerical Study of the Dynamic, Thermodynamic and Microstructural Parameters of Convective Clouds. Natural Science 10: 63-69. (doi: 10.4236/ns.2018.102006.).

Shmeter S.M., 1987, Thermodynamics and physics of convective clouds. Gidrometeoizdat, Leningrad.

Stephens G.L., Christensen M., Andrews T., Haywood J., Malavelle F.F., Suzuki K. Jing X., Lebsock M., Li J.-L.F. \& Ousmane Sy H.T., 2019, Cloud Physics from Space. Quarterly Journal of the Royal Meteorological Society. (https://doi.org/10.1002/qj.3589).

Straka J.M., 2009, Cloud and precipitation microphysics, Principles and Parameterizations. Cambridge University Press, Cambridge,

UCP, 2019, Understanding Clouds and Precipitation. Conference Proceedings, Harnack-Haus (Max-Planck-Institut für Meterorologie), Berlin, February 25- March 1 2019. (https://indico.mpimet.mpg.de/event/1/).

Veremei N.E., Dovgaluk Yu.A., Zatevakhin M.A., Ignatiev A.A., Morozov V.N. \& Pastushkov R.S., 2016, Evaluation of water resources available for active modifications of convective clouds by means of hygroscopical reagent. Proceedings of A.I. Voeikov Main Geophysical Observatory 582: 45-91.

Vladimirov S.A. \& Pastushkov R.S., 2016, The complex method of convective cloud seeding for purposes to regulate precipitation. Three-dimensional numerical simulation. Proceedings of A.I. Voeikov Main Geophysical Observatory 582: 116-127. 